SELECCIONES MATEMÁTICAS
Universidad Nacional de Trujillo
ISSN: 2411-1783 (Online)
2019; Vol.06(2): $189-195$.

\title{
Optimal Control For Malaria Epidemic Model With Vaccinating, Human Treatment And Mosquitos Spraying.
}

\author{
Imam Fahcruddin* (iD, Salmah Salmah. ${ }^{\dagger}$ (i) , and April Gunawan Malau. ${ }^{\ddagger(\mathbb{D})}$
}

Received, Aug. 20, 2019

Accepted, Nov. 28, 2019

How to cite this article:

Fahcruddin, I., et al. Optimal Control For Malaria Epidemic Model With Vaccinating, Human Treatment And Mosquitos Spraying. Selecciones Matemáticas. 2019; 6(2):189-195. http://dx.doi .org/10.17268/sel.mat.2019.02.05

\begin{abstract}
In this paper we study the effect of vaccination control, medical treatment and spraying to malaria epidemic model. Firstly the non-control malaria epidemic model is generated and the equilibrium point is determined. Afterward, the stability of equilibrium point in previous model is investigated. The research is continued by deciding the optimal control of malaria epidemic model and minimizing the cost. The results show that the control effect can reduce the subpopulation of infected human and mosquitoes.
\end{abstract}

Keywords. Malaria epidemic model, Stability, Equilibrium point, Control optimal.

1. Introduction. Malaria is one of the infectious diseases in which the control efforts to be a global commitment to the Millennium Development Goals (MDGs) [9,10,12-14]. Malaria is caused by Plasmodium parasites that live and breed in human red blood cells that are transmitted by Anopheles female mosquitoes, which can affect all people, both men and women in all age groups from babies, children and adults [3,24-28,30]. Approximately $80 \%$ of districts or cities in Indonesia including the category of malaria-endemic areas and more than $45 \%$ of Indonesia's population live in malaria-endemic areas [17]. Several attempts were made to prevent the spread of malaria such as giving vaccines and treatment among the human population and also spraying the mosquito population.

From several models of controlling the spread of diseases that involve the mosquito population and human population, the control strategy is focused on mosquito populations, such results from Thom, et al [28] which discusses the application of optimal control to prevent the spread of dengue fever with sterilization control insects and insecticides in the mosquito Aedes aegypti. Sachs [27] states that efforts to eradicate the disease is not enough to eliminate the mosquito as its medium spread of the disease. However, control strategies involving the control of the human population also need to be involved in mathematical modeling, such as results by Makinde, et al [18] that discusses optimal control models to control malaria epidemic by quarantine and treatment in humans as well as spraying the mosquitoes. Then, Okosun [22] examined the optimal control of malaria epidemic models with vaccination control and treatment in humans.

From the research of Makinde [18], the quarantine is done by separating the latent and the vulnerable infected population so as to prevent and treat human that are infected with malaria. Then, the control from vaccination is not applied to the studied model, whereas the research on vaccination to prevent malaria epidemic is growing rapidly. In Okosun [22], the reduction of intervention, such as spraying using chemicals to eradicate the mosquitoes are not applied in the model under study. In this paper, it is discussed the model of epidemic malaria control vaccination and treatment in humans, such as spraying the mosquito population that has not been discussed in the previous

\footnotetext{
*Engine Department, Sekolah Tinggi Ilmu Pelayaran Jakarta, Indonesia (fahrudinuin@gmai l . com),

$\dagger$ Mathematics Department, Universitas Gadjah Mada, Indonesia (syalmah@yahoo . com),

${ }^{\ddagger}$ Port and Shipping Department, Sekolah Tinggi Ilmu Pelayaran Jakarta, Indonesia (apri lgunawan22@gma i 1 . com).
} 
paper. Control that using vaccination and treatment applied to human populations to reduce the number of patients with malaria and deaths from malaria. Then spraying with chemicals applied to reduce the mosquito population, especially mosquitoes that infected with malaria. In addition, the cost of implementing the control aspect is also minimized so the efforts to eradicate malaria done efficiently and optimally.

2. Malaria Epidemic Model.. The model sub-divides the total human population [5,7], denoted by $N_{h}$, into sub-populations of susceptible individuals $\left(S_{h}\right)$, those exposed to malaria $\left(E_{h}\right)$, individuals with malaria symtoms $\left(I_{h}\right)$, recovered human $\left(R_{h}\right)$ and vaccinated individuals $\left(V_{h}\right)$. So that $N_{h}=S_{h}+E_{h}+I_{h}+R_{h}+V_{h}$.

The total vector (mosquito) population, denoted by $N_{v}$, is subdivided into susceptible mosquitoes $\left(S_{v}\right)$, mosquitoes exposed to the malaria parasite $\left(E_{v}\right)$ and infectious mosquitoes $\left(I_{v}\right)$. Thus, $N_{v}=S_{v}+E_{v}+I_{v}$.

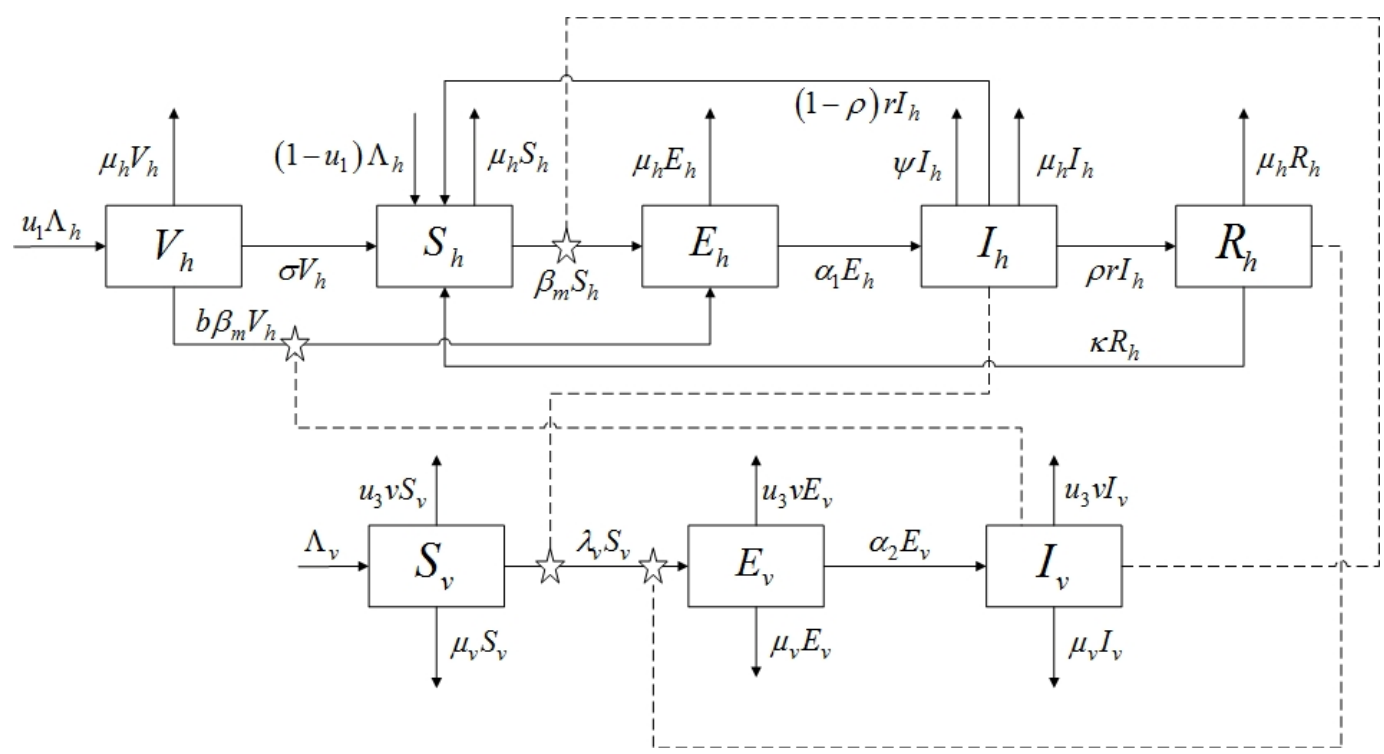

FIGURE 2.1. Flow diagram for malaria disease transmission

The model is given by the following system of ordinary differential equations:

$$
\begin{aligned}
\frac{d}{d t} V_{h}(t) & =u_{1}(t) \Lambda_{h}-\left(\mu_{h}+\sigma+\lambda_{h}(t)\right) V_{h}(t) \\
\frac{d}{d t} S_{h}(t) & =\left(1-u_{1}(t)\right) \Lambda_{h}+\kappa R_{h}(t)-\lambda_{h}(t) S_{h}(t)+\sigma V_{h}(t)-\mu_{h} S_{h}(t)+\left(\theta+\tau u_{2}(t)\right)(1-\rho) I_{h}(t) \\
\frac{d}{d t} E_{h}(t) & =\lambda_{h}(t) S_{h}(t)+b \lambda_{h}(t) V_{h}(t)-\left(\alpha_{1}+\mu_{h}\right) E_{h}(t) \\
\frac{d}{d t} I_{h}(t) & =\alpha_{1} E_{h}(t)-\left(\theta+\tau u_{2}(t)+\psi+\mu_{h}\right) I_{h}(t) \\
\frac{d}{d t} R_{h}(t) & =\left(\theta+\tau u_{2}(t)\right) \rho I_{h}(t)-\left(\kappa+\mu_{h}\right) R_{h}(t) \\
\frac{d}{d t} S_{v}(t) & =\Lambda_{v}-\left(\lambda_{v}(t)+\mu_{v}+u_{3}(t) v\right) S_{v}(t) \\
\frac{d}{d t} E_{v}(t) & =\lambda_{v}(t) S_{v}-\left(\alpha_{2}+\mu_{v}+u_{3}(t) v\right) E_{v}(t) \\
\frac{d}{d t} I_{v}(t) & =\alpha_{2} E_{v}-\left(\mu_{v}+u_{3}(t) v\right) I_{v}(t)
\end{aligned}
$$

with $\lambda_{h}(t)=\beta \epsilon \phi I_{v}(t), \lambda_{v}(t)=\lambda \epsilon \phi\left(I_{h}(t)+\eta R_{h}(t)\right)$ and initial condition

$$
\begin{array}{rlrl}
V_{h}(0) & =V_{h 0}>0, S_{h}(0)=S_{h 0}>0, & & E_{h}(0)=E_{h 0}>0, I_{h}(0)=I_{h 0}>0, \\
R_{h}(0)=R_{h 0}>0, S_{v}(0) & =S_{v 0}>0, & E_{v}(0)=E_{v 0}>0 \text { and } I_{v}(0)=I_{v 0}>0
\end{array}
$$

Susceptible individuals are recruited at a rate $\Lambda_{h}$ where a proportion $u_{1} \in[0,1]$ of them is successfully vaccinated at birth. Susceptible individuals acquire malaria through contact with infectious mosquitoes at a rate $\lambda_{v}(t)$. Due to waning effect, some vaccinated individuals will move to the exposed class at a rate $b \lambda_{v}(t)$, where $(1-b) \in[0,1]$ is the efficacy of vaccine or they loose their immunity completely and move to the susceptible class at a rate $\sigma$. Exposed individuals move to the infectious class at a rate $\alpha_{1}$. Individuals with malaria are treated 
under control, at a rate $\tau u_{2}(t), \theta$ are individuals who recovered spontaneously. A proportion of them, $\rho$, moves to the recovered class with temporary immunity and the other proportion moves to the susceptible class. Non treated infected individuals die at a rate $\psi$. Recovered individual loose immunity at a rate $\kappa$ and become susceptible again. The term $\mu_{h}$ is the natural death rate.

Susceptible mosquitoes $\left(S_{v}\right)$ are generated at a rate $\Lambda_{v}$ and acquire malaria through contacts with infected humans at a rate $\lambda_{v}(t)$. Mosquitoes are assumed to suffer death due to natural causes at a rate $\mu_{v}$. Newly infected mosquitoes move to the exposed class $\left(E_{v}\right)$, and later progress to the class of symptomatic mosquitoes $\left(I_{v}\right)$ at a rate $\alpha_{2}$. Mosquitoes deaths due to spraying at a rate $u_{3}(t) v$. We also consider two forms of infection for mosquitoes. Here $\lambda_{h}(t)=\beta \epsilon \phi I_{v}(t)$ and $\lambda_{v}(t)=\lambda \epsilon \phi\left(I_{h}(t)+\eta R_{h}(t)\right)$, where $\beta$ is the transmission probability per bite, $\epsilon$ is the per capita biting rate of mosquitoes and $\phi$ is the contact rate of vector per human per unit time. The terms $\lambda$ and $\eta$ are the probability for a vector to get infected by an infectious human and modificationn parameter, respectively. Further, using Theorem 2 in Van den Driessche and Watmough [31], the following result is established.

3. Basic Results.. In this section, we study some basic results of the solution of the system (2.1-2.9) which will be very useful to use into proof of stability and persistence results.

$$
\begin{aligned}
& \text { Theorem 3.1. Let } \mathcal{R}_{0}=\sqrt{\frac{\lambda \epsilon^{2} \phi^{2} \Lambda_{h} \Lambda_{v} \alpha_{1} \alpha_{2} \beta\left(k_{3}+\eta \theta\right)}{\mu_{h} \mu_{v}^{2} k_{1} k_{2} k_{3}}} \text { with } k_{1}=\left(\alpha_{1}+\mu_{h}\right), k_{2}=\left(\theta+\psi+\mu_{h}\right) \text {, } \\
& k_{3}=\left(\kappa+\mu_{h}\right) \text { and } k_{4}=\left(\alpha_{2}+\mu_{v}\right) \text {. } \\
& \quad i \text { If } \mathcal{R}_{0} \leq 1 \text { then exist one disease free equilibrium point } x_{0}=\left(\frac{\Lambda_{h}}{\mu_{h}}, 0,0,0, \frac{\Lambda_{v}}{\mu_{v}}, 0,0\right) \text {. } \\
& \quad \text { ii If } \mathcal{R}_{0}>1 \text { then exist disease free equilibrium point } x_{0} \text { and one equilibrium endemic }
\end{aligned}
$$

$$
x_{e}=\left(S_{h}^{\star}, E_{h}^{\star}, I_{h}^{\star}, R_{h}^{\star}, S_{v}^{\star}, E_{v}^{\star}, I_{v}^{\star}\right)
$$

with

$$
\begin{aligned}
& a_{0}=k_{4} \mu_{v}\left(\lambda \epsilon \phi \Lambda_{h} \alpha_{1}\left(k_{3}+\eta \theta\right)+\mu_{v}\left(k_{1} k_{2} k_{3}-\kappa \alpha_{1} \theta\right)\right) \text {, } \\
& b_{0}=\mu_{h} \mu_{v}^{2} k_{1} k_{2} k_{3} k_{4}\left(1-\mathcal{R}_{0}^{2}\right) \text {, } \\
& S_{h}^{\star}=\frac{\Lambda_{h} k_{1} k_{2} k_{3}}{\left(\mu_{h}-\frac{b_{0}}{a_{0}}\right) k_{1} k_{2} k_{3}+\frac{\kappa \alpha_{1} \theta b_{0}}{a_{0}}}, \\
& I_{h}^{\star}=\frac{\tilde{\lambda_{h}} \Lambda_{h} k_{3} \alpha_{1}}{\left(\mu_{h}-\frac{b_{0}}{a_{0}}\right) k_{1} k_{2} k_{3}+\frac{\kappa \alpha_{1} \theta b_{0}}{a_{0}}} \\
& S_{v}^{\star}=\frac{\Lambda_{v}}{\left(\lambda \epsilon \phi\left(I_{h}^{\star}+\eta R_{h}^{\star}\right)+\mu_{v}\right)} \\
& I_{v}^{\star}=\frac{\alpha_{2} \tilde{\lambda_{v}} \Lambda_{v}}{k_{4} \mu_{v}\left(\lambda \epsilon \phi\left(I_{h}^{\star}+\eta R_{h}^{\star}\right)+\mu_{v}\right)} \\
& \begin{aligned}
E_{h}^{\star} & =\frac{\Lambda_{h} k_{1} k_{2} k_{3}}{\left(\mu_{h}-\frac{b_{0}}{a_{0}}\right) k_{1} k_{2} k_{3}+\frac{\kappa \alpha_{1} \theta b_{0}}{a_{0}}} \\
R_{h}^{\star} & =\frac{\theta \tilde{\lambda_{h}} \Lambda_{h} \alpha_{1}}{\left(\mu_{h}-\frac{b_{0}}{a_{0}}\right) k_{1} k_{2} k_{3}+\frac{\kappa \alpha_{1} \theta b_{0}}{a_{0}}} \\
E_{v}^{\star} & =\frac{\tilde{\lambda_{v}} \Lambda_{v}}{k_{4}\left(\lambda \epsilon \phi\left(I_{h}^{\star}+\eta R_{h}^{\star}\right)+\mu_{v}\right)}
\end{aligned}
\end{aligned}
$$

Stability analytic of disease free equilibrium point [8,32] given by Theorem 3.2.

Theorem 3.2. if $\mathcal{R}_{0}<1$ then disease free equilibrium $x_{0}$ locally asymptotically stable.

Besides to applying controls to reduce human population and mosquitoes infected with malaria, the cost aspect caused by malaria also need to be minimized. Cost is made up of the cost of implementing controls, including the average of the cost of implementing the vaccine $(n)$, treatment $(c)$ and spraying $(d)$. Then, it is also covered the average amount of loss incurred by malaria-infected human subpopulation $(m)$.

In designing a dynamic cost function, it involves the time variable associated with the specified planning period. It is assumed that the function of the controls is in the form of a quadratic cost function. If the established planning period of the cost allocated to tackle malaria is $\left[0, t_{1}\right]$ and the nominal interest rate is $q$, then the cost function model of epidemic malaria is a particular integral of the multiplication disconting factor with the sum of the rate of change in implementing cost control and cost of losses borne by people infected with malaria, so that $[1,6]$ :

$$
J\left(u_{1}, u_{2}, u_{3}\right)=\int_{0}^{t_{1}} e^{-q t}\left(m I_{h}(t)+n u_{1}^{2}(t)+c u_{2}^{2}(t)+d u_{3}^{2}(t)\right) d t .
$$

Our purpose is to find an optimal control pair $u_{1}^{\star}(t), u_{2}^{\star}(t)$ and $u_{3}^{\star}(t)$, such that

$$
J\left(u_{1}^{\star}(t), u_{2}^{\star}(t), u_{3}^{\star}(t)\right)=\min _{\omega} J\left(u_{1}, u_{2}, u_{3}\right)
$$


where $\omega=\left\{\left(u_{1}, u_{2}, u_{3}\right) \in L^{1}\left(0, t_{1}\right) \mid u_{1} \in[0,1], \forall i=1,2,3\right\}$ are fixed nonnegative constants and $\left(u_{1}, u_{2}, u_{3}\right) \in$ $L^{1}\left(0, t_{1}\right)$ means that $u_{1}, u_{2}, u_{3}$ is Lebesgue measurable on $\left(0, t_{1}\right)$.

The Hamiltonian function $\mathcal{H}$ with respect to $u_{1}, u_{2}, u_{3}$ is defined as following

$$
\begin{aligned}
\mathcal{H}_{c}=\left(a I_{h}\right. & \left.+n u_{1}^{2}+c u_{2}^{2}+d u_{3}^{2}\right) \\
& +m_{s_{h}}\left(\left(1-u_{1}\right) \Lambda_{h}+\kappa R_{h}-\beta \epsilon \phi I_{v} S_{h}+\sigma V_{h}-\mu_{h} S_{h}+\left(\theta+\tau u_{2}\right)(1-\rho) I_{h}\right) \\
& +m_{E_{h}}\left(\beta \epsilon \phi I_{v} S_{h}+b \beta \epsilon \phi I_{v} V_{h}-\left(\alpha_{1}+\mu_{h}\right) E_{h}\right) \\
& +m_{I_{h}}\left(\alpha_{1} E_{h}-\left(\theta+\tau u_{2}+\psi+\mu_{h}\right) I_{h}\right)+m_{R_{h}}\left(\left(\theta+\tau u_{2}\right) \rho I_{h}-\left(\kappa+\mu_{h}\right) R_{h}\right) \\
& +m_{V_{h}}\left(u_{1} \Lambda_{h}-\left(\mu_{h}+\sigma+b \beta \phi I_{v}\right) V_{h}\right) \\
& +m_{S_{v}}\left(\Lambda_{v}-\left(\beta \epsilon \phi\left(S_{h}+b V_{h}\right)+\mu_{v}+u_{3} v\right) S_{v}\right) \\
& +m_{E_{v}}\left(\beta \epsilon \phi\left(S_{h}+b V_{h}\right) S_{v}-\left(\alpha_{2}+\mu_{v}+u_{3} v\right) E_{v}\right) \\
& +m_{I_{v}}(t)\left(\alpha_{2} E_{v}-\left(\mu_{v}+u_{3} v\right) I_{v}\right)
\end{aligned}
$$

with costate equation is

$$
\begin{aligned}
\frac{d m_{V_{h}}}{d t} & =-\sigma m_{S_{h}}+\left(\sigma+\mu_{h}\right) m_{V_{h}}+\left(m_{V_{h}}-m_{E_{h}}\right) b \beta \epsilon \phi I_{v}+q m_{V_{h}} \\
\frac{d m_{S_{h}}}{d t} & =\left(-\beta \epsilon \phi I_{v}-\mu_{h}\right) m_{S_{h}}+\left(\beta \epsilon \phi I_{v}\right) m_{E_{h}}+q m_{S_{h}} \\
\frac{d m_{E_{h}}}{d t} & =\left(\mu_{h}+\alpha_{1}\right) m_{E_{h}}-\alpha_{1} m_{I_{h}}+q m_{E h} \\
\frac{d m_{I_{h}}}{d t} & =\left(\theta+\tau u_{2}+\psi+\mu_{h}\right) m_{I_{h}}-\rho\left(\theta+\tau u_{2}\right) m_{R_{h}}-(1-\rho)\left(\theta+\tau u_{2}\right) m_{S_{h}}+\left(\lambda \epsilon \phi S_{v}\right) m_{S_{v}} \\
& +\lambda \epsilon \phi S_{v} m_{E_{v}}+q m_{I_{h}} \\
\frac{d m_{R_{h}}}{d t} & =-\kappa m_{S_{h}}+\left(\mu_{h}+\kappa\right) m_{R_{h}}+\lambda \epsilon \phi \eta S_{v}\left(m_{S_{v}}-m_{E_{v}}\right)+q m_{R_{h}} \\
\frac{d m_{S_{v}}}{d t} & =\left(\lambda \epsilon \phi\left(I_{h}+\eta R_{h}\right)+\mu_{v}\right) m_{S_{v}}-\lambda \epsilon \phi\left(I_{h}+\eta R_{h}\right) m_{E_{v}}+q m_{S_{v}} \\
\frac{d m_{E_{v}}}{d t} & =\left(\alpha_{2}+\mu_{v}\right) m_{E_{v}}-\alpha_{2} m_{I_{v}}+q m_{E_{v}} \\
\frac{d m_{I_{v}}}{d t} & =\left(\beta \epsilon \phi S_{h}\right) m_{S_{h}}-m_{E_{h}}\left(\beta \epsilon \phi\left(S_{h}+b V_{h}\right)\right)+\mu_{v} m_{I_{v}}+b \beta \epsilon \phi V_{h} m_{V_{h}}
\end{aligned}
$$

By applying Hamiltonian method, we can obtain the following necessary conditions that a pair of optimal controls and corresponding states must satisfy.

Theorem 3.3. There exist optimal control $\left(u_{1}^{\star}(t), u_{2}^{\star}(t), u_{3}^{\star}(t)\right)$ that minimizes (3.1) with constrain (3.4)-(3.11) is

$$
\begin{aligned}
& u_{1}^{\star}=\max \left\{0, \min \left(1, \frac{\left(m_{S_{h}}-m_{V_{h}}\right) \Lambda_{h}}{2 n}\right)\right\}, \\
& u_{2}^{\star}=\max \left\{0, \min \left(1, \frac{\tau\left(m_{I_{h}}-\rho m_{R_{h}}-(1-\rho) m_{S_{h}}\right) I_{h}}{2 c}\right)\right\}, \\
& u_{3}^{\star}=\max \left\{0, \min \left(1, \frac{\left(m_{S_{v}} S_{v}+m_{E_{v}} E_{v}+m_{I_{v}} I_{v}\right) v}{2 d}\right)\right\},
\end{aligned}
$$

with $m_{V_{h}}(t), m_{S_{h}}(t), m_{E_{h}}(t), m_{I_{h}}(t), m_{R_{h}}(t), m_{S_{v}}(t), m_{E_{v}}(t)$ and $m_{I_{v}}(t)$ is solution of equation (18) - (25) that transversal condition $m_{V_{h}}\left(t_{1}\right)=m_{S_{h}}\left(t_{1}\right)=m_{E_{h}}\left(t_{1}\right)=m_{I_{h}}\left(t_{1}\right)=m_{R_{h}}\left(t_{1}\right)=m_{S_{v}}\left(t_{1}\right)=m_{E_{v}}\left(t_{1}\right)=$ $m_{I_{v}}\left(t_{1}\right)=0$

The following will be simulated about the epidemic models with control malaria vaccination and treatment of human and mosquito spraying. To determine how much the influence of the controls provided in reducing human subpopulations and malaria-infected mosquitoes, the graph of the simulation consists of 4 graphs, namely:

i using vaccination $\left(u_{1}\right)$ without insecticide spraying $\left(u_{3}=0\right)$ and no treatment of the symptomatic humans $\left(u_{2}=0\right)$,

ii treating the symptomatic humans $\left(u_{2}\right)$ without using insecticide spraying $\left(u_{3}=0\right)$ and no vaccination $\left(u_{1}=0\right)$,

iii using insecticide spraying $\left(u_{3}\right)$ without vaccination $\left(u_{1}=0\right)$ and no treatment of the symptomatic humans $\left(u_{2}=0\right)$,

iv using all three control measures $\left(u_{1}, u_{2}, u_{3}\right)$. 
TABLE 3.1

Description of Variables and Parameters of the Malaria Model [18, 22]

\begin{tabular}{clcl}
\hline Notation & Value & Notation & Value \\
\hline$\Lambda_{h}$ & 99/ day & $\theta$ & 0.005 \\
$\mu_{h}$ & $0.0000421 /$ day & $\Lambda_{v}$ & $890 /$ day \\
$\lambda$ & 0.0057233 & $\mu_{v}$ & $0.05 /$ day \\
$\alpha_{2}$ & $0.0556 /$ day & $\eta$ & 0.001 \\
$\kappa$ & 0.7902 /day & $b$ & 0.03 \\
$\psi$ & 0.02 /day & $\sigma$ & $0.005 /$ day \\
$\alpha_{1}$ & 0.0588 /day & $C$ & US\$ 500 \\
$\alpha_{2}$ & $0.0556 /$ day & $D$ & US $\$ 50$ \\
$v$ & 0.6 & $\tau$ & 0.7 \\
$m$ & US\$ 150 & $\rho$ & $0.023 /$ day \\
$n$ & US\$ 100 & $v$ & 0.6 \\
\hline
\end{tabular}

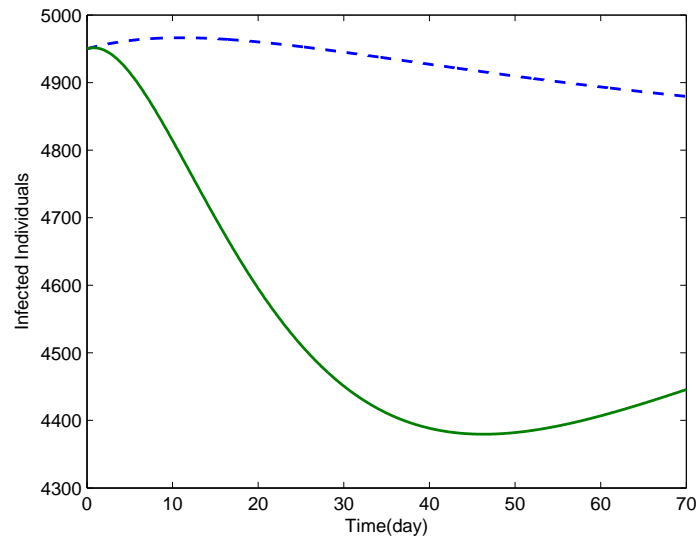

(a)

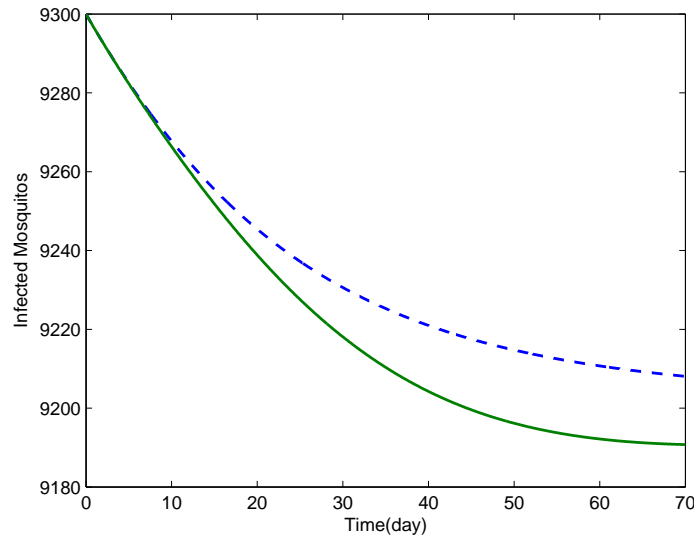

(b)

FIGURE 3.1. Dynamical behavior when $u_{1} \neq 0, u_{2}=u_{3}=0$ for (a) infected human population (b) infected mosquitoes population, where the straight line indicates for dynamic with vaccination and the dashed line without vaccination control.

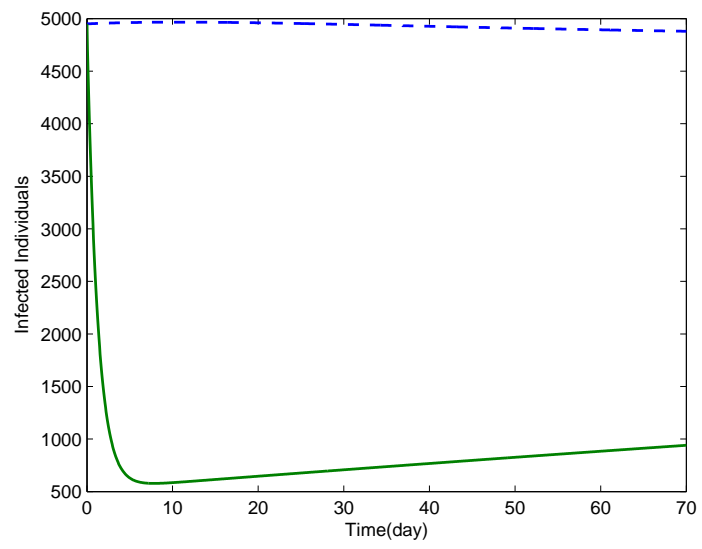

(a)

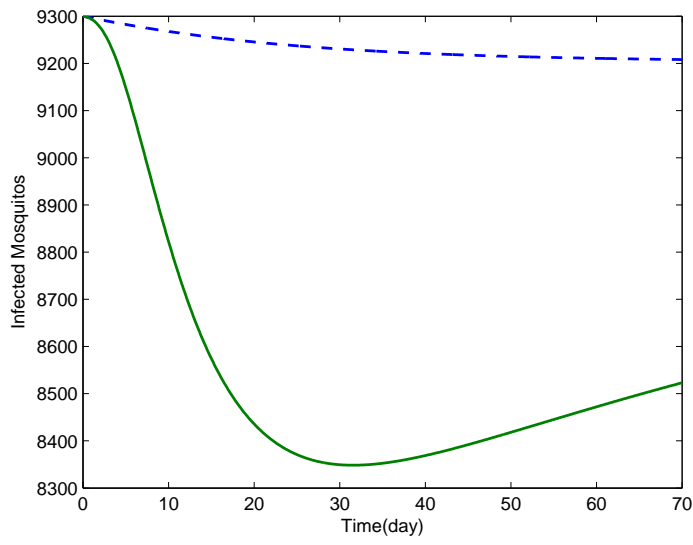

(b)

FIGURE 3.2. Dynamical behavior when $u_{2} \neq 0, u_{1}=u_{3}=0$ for (a) infected human population (b) infected mosquitoes population, where the straight line indicates for dynamic with treatment and the dashed line without treatment control.

Furthermore, simulation results obtained from the graph with Forward-Backward Sweep Runge-Kutta [2] showing the relation number of mosquitoes susceptible subpopulations.

In Figure 3.1-3.3 shows that the number of humans and mosquitoes infected by malaria that is applying one of the control fewer than the number of humans and mosquitoes infected by malaria without control. This indicates that by applying one of the models of epidemic malaria control can reduce the number of infected humans and 
mosquitoes in malaria epidemic models without control.

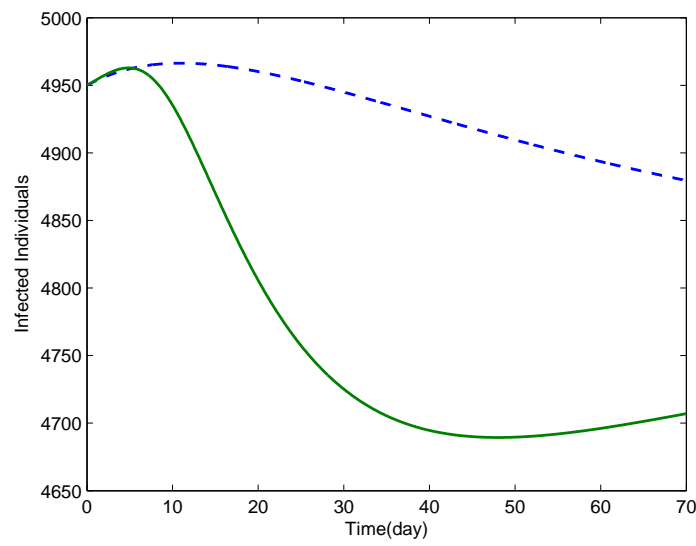

(a)

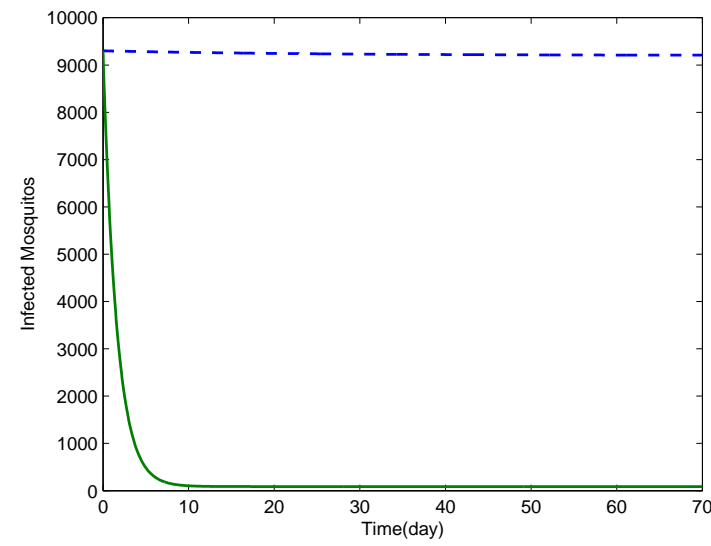

(b)

FIGURE 3.3. Dynamical behavior when $u_{3} \neq 0, u_{1}=u_{2}=0$ for (a) infected human population (b) infected mosquitoes population, where the straight line indicates for dynamic with spraying and the dashed line without spraying.

In Figure 3.4 shows that the number of humans and mosquitoes infected by malaria that applying three controls at once fewer than the number of humans and mosquitoes infected with malaria without control even with one or two controls are applied. This indicates that the results of numerical simulation shows that by applying the three controls at once, namely vaccination, treatment and spraying are more optimal to reduce the number of subpopulations of human and malaria-infected mosquitoes.

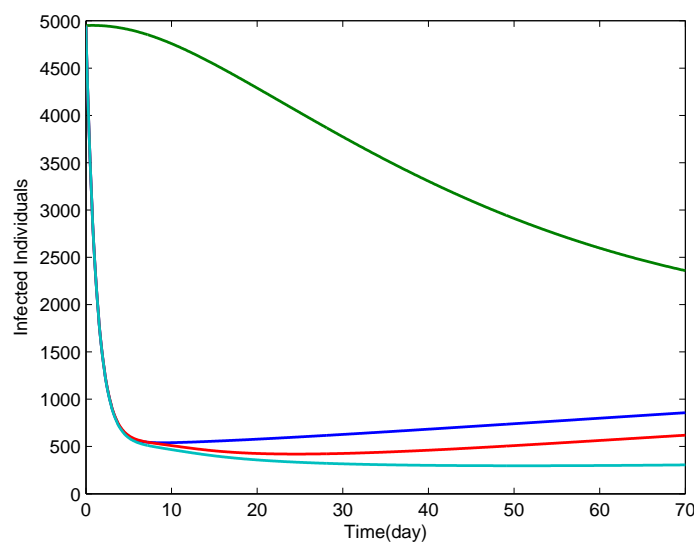

(a)

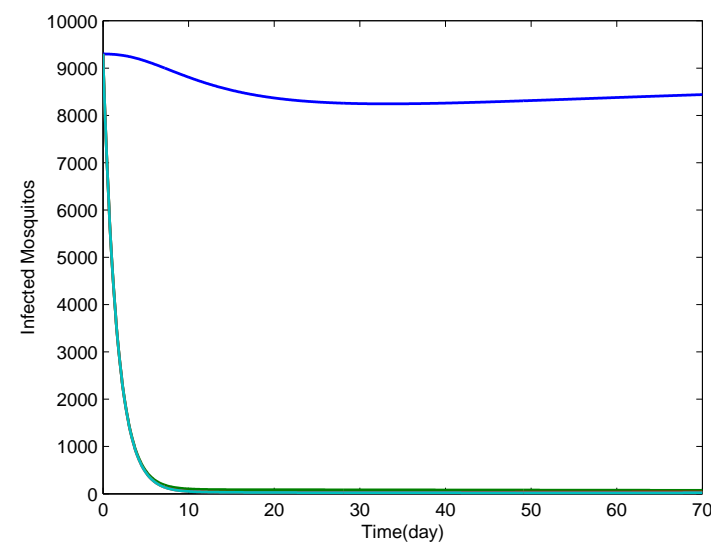

(b)

FIGURE 3.4. Dynamical behavior for (a) infected human population (b) infected mosquitoes population; blue color straight line indicates behavior when $u_{1} \neq 0$ and $u_{2} \neq 0$; green color straight line indicates behavior when $u_{1} \neq 0$ and $u_{3} \neq 0$; red color straight line indicates behavior when $u_{2} \neq 0$ and $u_{3} \neq 0$; black color straight line indicates behavior when $u_{1} \neq 0, u_{2} \neq 0$, and $u_{3} \neq 0$

4. Conclusions. In this paper discussed the model of malaria epidemics involving vaccination and treatment of the human population as well as spraying the mosquito population. By determining the number base reproduction $\left(\mathcal{R}_{0}\right)$, the existence and the stability of the equilibrium point of malaria epidemic models without control can be analyzed. Furthermore, when $\mathcal{R}_{0}<1$ indicates that the incidence of malaria in the area of malaria will be lost for a long time.

The next discussion is to determine the optimal control of epidemic malaria models and minimize the cost of implementing controls. Numerical simulation results indicate that the effect of control given to the endemic and non-endemic malaria can reduce the number of subpopulations of human and malaria-infected mosquitoes.

In this paper, the controls that applied are vaccination and treatment of human and mosquito spraying. Then the stability of the equilibrium point that studied is the local stability of the disease-free equilibrium point. This research can be deeper by examining the global stability of disease-free equilibrium and endemic malaria in epidemic models and apply other forms of such control that can reduce human subpopulations and mosquitoes infected with malaria near to zero in malaria endemic areas. 
ORCID and License

Imam Fahcruddin https://orcid.org/0000-0003-4976-7221,

Salmah Salmah https://orcid.org/0000-0002-5774-4321,

April Gunawan Malau https://orcid.org/0000-0001-9444-7599.

This work is licensed under the Creative Commons Attribution-NoComercial-ShareAlike 4.0.

\section{References}

[1] Bazaraa, M. S. dan Shetty, C. M., Nonlinear Programming. John Wiley \& Sons, Inc, 1993.

[2] Bradie, B. A Friendly Introduction to Numerical Analysis, Prentice Hall, New Jersey, USA, 2006.

[3] Chiang, Alpha Fundamental Methods of Mathematical Economics, McGraw-Hill, New York, USA, 1927.

[4] Chiang, Alpha Elements of Dynamic Optimization, McGraw-Hill, New York, USA, 992.

[5] Chitnis, N., Cushing, J. M., \& Hyman, J. M. Bifurcation analysis of a mathematical model for malaria transmission. SIAM Journal on Applied Mathematics, 2006; 67(1):24-45.

[6] Chong, E. K. P. and Zak, S. H. An Introduction to Optimization, John Wiley and Sons, Inc. 1996.

[7] Ceuser, W and Smith, B, Malaria Diagnosis and the Plasmodium Life Nature Procedings, 2009: doi:10.1038/npre.2009.3960.1, New York. : Posted 7 Nov 2009.

[8] Diekmann, O. and Heesterberk, J.A.P. Mathematical Epidemiology of Infectious Diseases: Model Building, Analysis and Interpretation, John Wiley and Sons, Chichester, 2000.

[9] Foster WA, Walker ED. Mosquitoes (Culicidae). In Mullen G, Durden L, eds, Medical and Veterinary Entomology, Academic Press, San Deigo, 2002.

[10] Macdonald, G. The epidemiology and control of malaria, Oxford UniversityPress, London, 1957.

[11] Gopal, M. Modern Control System Theory, Wiley Eastern Limited, New Delhi, 1984.

[12] Gilles, HM Peter, F.B. Rationale and Technique of malaria control.: Essential Malariology, Arnold, Oxford University Press, New York, 2002.

[13] Yang, H. M. Malaria transmission model for different levels of acquired immunity and temperature-dependent parameters (vector), Revista de Saude Publica, ,2000; 34:223-231.

[14] Harijanto PN. Malaria: Epidemiologi, Patogenesis, Manifestasi Klinis dan penanganannya, EGC, Jakarta, 2000.

[15] Khalil, H.K. Nonlinear Systems, 3rd edition, Prentice Hall, New Jersey, USA, 2002.

[16] Koella, J. C. On the use of mathematical models of malaria transmission, Acta Tropica, 1991; 49:1-25.

[17] Kemenkes RI. Profil Kesehatan Indonesia 2010, Kementrian Kesehatan Republik Indonesia, Jakarta, 2011.

[18] Makinde, O.D., Okosun, K.O. Impact of chemo-theraphy on optimal control of malaria disease with infected immigrants. Biosystems, 2011; 104:32-41.

[19] Mital, K.V. Optimization Methods in Operation Research and Systems Analysis, Wiley Eastern Limited, New Delhi, 1984.

[20] Nedelman, J. Introductory review: Some new thoughts about some old malaria models, J. Math .Biosci. , 1985; 73:159-182.

[21] Olsder, G.J. Mathematical Systems Theory, Delftse Uitgevers Maatschappij, CW Delft, Netherlands, 1994.

[22] Okosun, K.O. Optimal Control Analysis of a malaria disease transmission model that includes treatment and vaccination with maning immunity. Biosystems, 2011; 106:136 - 145 .

[23] Perko, S. Differential Equations and Dynamical Systems, Texts in Applied Mathematics, Springer-Verlag, USA, 1991.

[24] Ross, R. The prevention of malaria, John Murray, London, 1911.

[25] Rinidar dan Mawengkang, M. Modeling the dynamics of malaria-mosquito-human interactions as a way to control the outbreak, Proceeding. International Conference on Environmental research and technology (ICERT), Penang, Malaysia, 2008.

[26] Syaifudin, dkk. Pengembangan Vaksin Malaria dengan Radiasi Pengion, Prosiding Seminar Nasional Sains dan Teknologi-II, Universitas Lampung, 2008.

[27] Sachs, J. D. A new infected global effort to control malaria. Science, 2002: 298:122-124.

[28] Townson, The Anopheles Vector: Essential Malariology. Arnold Oxford University Press, New York, 2002.

[29] Thome, C.A. R., Yang, M. H., Esteva, L. Optimal Control of Aedes aegypti mosquitoes by the sterile insect technique and insecticide,J. Mathematical Bioscience, 2010; 223:12 - 23.

[30] Tu, Pierre, N.V. Dynamical System :An intoduction with application in Economis and Biology, Springer - Verlag, New-York, USA, 1994.

[31] Van den Driessche, P., dan Watmough, J. Reproduction numbers and sub-threshold endemic equilibria for comparmental models of disease transmission. J. Mathematical Biosciences, 2002; 180:29 - 48.

[32] Verhulst, F. Nonlinear Differential Equations and Dynamical Systems, Springer -Verlag, New- York, USA, 1990.

[33] WHO. World Report malaria, World Health Organization, Geneva, 2009. 